\title{
Constructing a Theory for Amorphous Solids
}

\section{Theorists are coming closer to a comprehensive description of the mechanics of solids with an amorphous structure, such as glass, cement, and compacted sand.}

\section{by Emanuela Del Gado*}

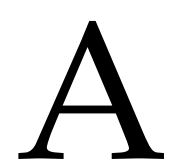
morphous solids are disordered assemblies of atoms or larger particles that nonetheless have a rigid structure. Examples include glass, cement, compacted sand, and even yogurt or chocolate mousse (Fig. 1). At first sight, these materials may appear to have little in common. But amorphous solids share many mechanical properties, and physicists have long been interested in finding a theory that predicts the materials' behavior in terms of their microstructure in a unified way. Unlike crystalline solids, however, the rigidity of amorphous solids is not associated with a thermodynamically stable, stressfree microstructure, so researchers have had to turn to novel theoretical approaches. A pair of papers from Eric DeGiuli of École Normale Supérieure in Paris [1, 2] now marks progress toward the goal of a unifying theory. Building on recent theories for glasses and granular materials, DeGiuli presents a

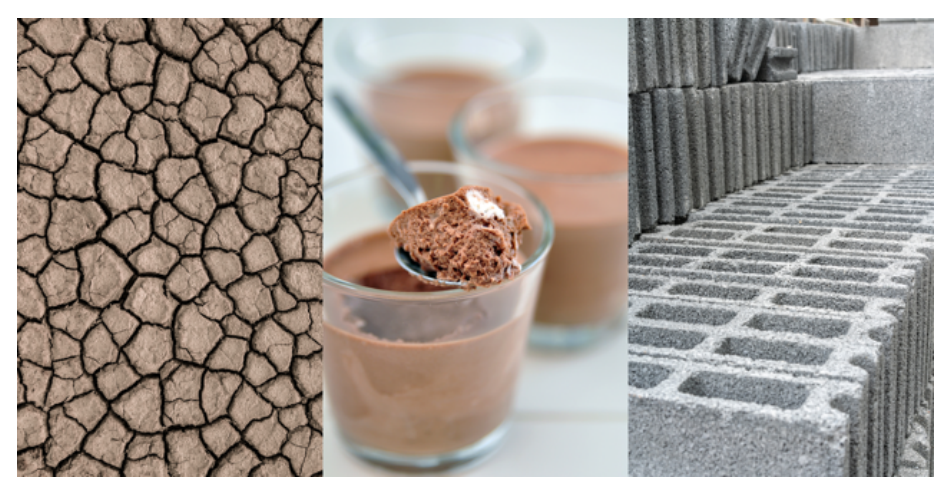

Figure 1: Compacted sand (left), chocolate mousse (middle), and concrete (right) are all types of amorphous solid-materials that are mechanically rigid but have a disordered structure. DeGiuli [1, 2] has developed a new theory that explains how stresses are correlated in these ubiquitous materials. (iStock.com/Vudhikul Ocharoen/themacx/A_Lein)

\footnotetext{
*Department of Physics, Georgetown University, Washington DC, USA
}

general field theory for amorphous solids and uses it to predict how they transmit stress.

Researchers are already equipped with equations that predict the mechanical response of a perfectly crystalline solid from its atomic structure [3]. These equations can, for example, describe how a piece of such a solid would deform under a load. But it has proven much harder to find an analogous set of equations for amorphous solids. The biggest barrier to developing such a theory is that, even in the absence of a load, an amorphous solid always has internal stresses, which build up within the material as it solidifies [4]. For example, when sand compacts, internal stresses develop from friction between the grains and from constraints imposed by the material's outer boundary. Depending on the material and how it's deformed, these internal stresses can lead to enhanced strength (think of the prestressed materials used for construction) or enhanced ductility [5, 6]. Measuring the distribution of internal stresses in experiments is very difficult. And even if one could measure it, the information would be incomplete: the stress distribution under a load evolves in a complicated way because stresses in different parts of the material are correlated. This correlation needs to be understood in order to predict how a material will deform when pushed.

An important theoretical breakthrough came in 2009 from Silke Henkes and Bulbul Chakraborty [7]. The duo adapted a nonequilibrium statistical mechanics approach that was developed by Sam Edwards in the 1980s to describe granular solids, a family of amorphous solids that includes compacted sand [8]. Following on Edwards' ideas, Henkes and Chakraborty defined a sand pile's macroscopic behavior in terms of its stress distribution. They then used their formulation to derive an equation for the stress correlation between two regions of the material that are separated by a distance $r$. The duo also sketched out a field theory to describe a 2D granular solid. This theory expresses the material's internal stresses with a mathematical field, the Airy stress function, whose properties are helpful for solving the equations that determine stress correlation.

DeGiuli's field theory [1, 2] builds on that of Henkes and Chakraborty, formulating it so that it applies not just to granular solids but to amorphous solids in general. He also 
derives the form of stress correlations in both 2D and 3D. Applied to a 2D material, the equations in his field theory predict that stress correlations in an amorphous solid are long-ranged, decaying as $1 / r^{2}$-just as Henkes and Chakraborty had shown for sand piles, and as another researcher, Anaël Lemaître, also demonstrated in 2017 for glasses [9]. In 3D, DeGiuli's equations predict stress correlations with a fall off of $1 / r^{3}$. Recently, Lemaître demonstrated the same result for glasses in 3D using a different approach [10].

Long-range stress correlations, like those DeGiuli and others have found in amorphous solids, are characteristic of an elastic solid - a material that bounces back to its original shape after being deformed. Such solids have traditionally been described with elasticity theory, which imagines that each small volume in a solid deforms elastically [11]. DeGiuli and the others $[1,2,7,9,10]$, however, don't make this assumption. Instead, they predict the same type of correlations by imposing two reasonable constraints on the stress field. First, the structure of the material is isotropic. Second, the net force and torque acting on each small volume in the material are zero (mechanical equilibrium).

The fact that these conditions are sufficient to lead to elastic-like correlations in an amorphous solid that is not necessarily elastic on small length scales is perhaps the most important insight from the new theoretical developments. For one, it provides a unifying explanation for why amorphous solids can, in many cases, resist deformation as do crystalline solids, despite not having a thermodynamically stable structure. Compacted sand is an interesting case in point: Once the sand settles into mechanical equilibrium, these theories argue that it will have solid-like properties despite there being no local elastic interactions between its grains-an effect that's seen in experiments. The same unifying principle could explain why so-called elastoplastic theories that include elasticity work extremely well for many types of amorphous materials [6]. And it is consistent with the observation that materials as diverse as glasses, cements, and granular matter all exhibit similar phenomena, such as avalanching, where a deformation in one point in the material triggers a chain of others, and strain localization, where the deformation resulting from pushing on a material doesn't spread out uniformly.

Field theories are powerful tools in condensed-matter and high-energy physics. And, in principle, the theory developed by DeGiuli could be used to do much more than predict stress correlations: It might, for example, be used to describe the response of amorphous solids to large defor- mations or to changing environmental conditions. But these additional calculations may be difficult because the theory is complex. Moreover, a field theory is a continuum theory-meaning it only applies at a length scale greater than the separation between the particles. Identifying this length scale and determining if DeGiuli's theory is truly distinct from existing continuum theories are important next steps. Resolving these issues will give researchers a better sense of whether they have found the right theory to connect the mechanics of amorphous solids with their microscopic complexity.

This research is published in Physical Review Letters and Physical Review E.

\section{REFERENCES}

[1] E. DeGiuli, "Field theory for amorphous solids," Phys. Rev. Lett. 121, 118001 (2018).

[2] E. DeGiuli, "Edwards field theory for glasses and granular matter," Phys. Rev. E 98, 033001 (2018).

[3] M. Born and K. Huang, Dynamical theory of crystal lattices (Oxford University Press, Oxford, 1954).

[4] S. Alexander, "Amorphous solids: Their structure, lattice dynamics and elasticity," Phys. Rep. 296, 65 (1998).

[5] T. C. Lubensky, C. L. Kane, X. Mao, A Souslov, and K. Sun, "Phonons and elasticity in critically coordinated lattices," Rep. Prog. Phys 78, 073901 (2015).

[6] A. Nicolas, E. E. Ferrero, K. Martens, and J. L. Barrat, "A review of mesoscale elastoplastic models," Rev. Mod. Phys. (to be published).

[7] S. Henkes and B. Chakraborty, "Statistical mechanics framework for static granular matter," Phys. Rev. E 79, 061301 (2009); G. Lois, J. Zhang, T. S. Majmudar, S. Henkes, B. Chakraborty, C. S. O'Hern, and R. P. Behringer, "Stress correlations in granular materials: An entropic formulation," Phys. Rev. E 80, 060303 (2009).

[8] S. F. Edwards and R. B. S. Oakeshott, "Theory of powders," Physica A 157, 1080 (1989); A. Mehta and S. F. Edwards, "Statistical mechanics of powder mixtures," Physica A 157, 1091 (1989).

[9] A. Lemaître, "Inherent stress correlations in a quiescent twodimensional liquid: Static analysis including finite-size effects," Phys. Rev. E 96, 052101 (2017).

[10] A. Lemaître, "Stress correlations in glasses," arXiv:1808.00994.

[11] L. D. Landau and E. M. Lifshitz, Theory of Elasticity, Course of Theoretical Physics Vol. 7 (Pergamon, Oxford, 1986).

10.1103/Physics. 11.88 